OPEN ACCESS

Edited by:

Nabiha Yusuf,

University of Alabama at Birmingham,

United States

Reviewed by:

Yu-Fang Shen,

Asia University, Taiwan

Yao Min Hung,

Kaohsiung Municipal United Hospital,

Taiwan

Wen-Yih Jeng,

National Cheng Kung University,

Taiwan

*Correspondence:

James Cheng-Chung Wei jccwei@gmail.com

Specialty section:

This article was submitted to Skin Cancer,

a section of the journal

Frontiers in Oncology

Received: 31 December 2021 Accepted: 03 February 2022

Published: 03 March 2022

Citation:

Chang $C-K$, Hsieh Y-S, Chen P-N, Chu S-C, Huang J-Y, Wang Y-H and Wei JC-C (2022) A

Cohort Study: Comorbidity and Stage Affected the Prognosis of Melanoma Patients in Taiwan.

Front. Oncol. 12:846760. doi: 10.3389/fonc.2022.846760

\section{A Cohort Study: Comorbidity and Stage Affected the Prognosis of Melanoma Patients in Taiwan}

\author{
Chin-Kuo Chang ${ }^{1}$, Yih-Shou Hsieh ${ }^{1,2}$, Pei-Ni Chen ${ }^{1,2}$, Shu-Chen $\mathrm{Chu}^{3}$, Jing-Yang Huang ${ }^{4}$, \\ Yu-Hsun Wang ${ }^{5}$ and James Cheng-Chung Wei ${ }^{1,6,7 *}$ \\ 1 Institute of Medicine, Chung Shan Medical University, Taichung, Taiwan, ${ }^{2}$ Department of Biochemistry, School of Medicine, \\ Chung Shan Medical University, Taichung, Taiwan, ${ }^{3}$ Institute and Department of Food Science, Central Taiwan University of \\ Science and Technology, Taichung, Taiwan, ${ }^{4}$ Center for Health Data Science, Chung Shan Medical University Hospital, \\ Taichung, Taiwan, ${ }^{5}$ Department of Medical Research, Chung Shan Medical University Hospital, Taichung, Taiwan, 6 Division \\ of Allergy, Immunology and Rheumatology, Chung Shan Medical University Hospital, Taichung, Taiwan, ${ }^{7}$ Graduate Institute \\ of Integrated Medicine, China Medical University, Taichung, Taiwan
}

Background: Comorbidities and stages may influence the prognosis of melanoma patients in Taiwan and need to be determined.

Methods: We performed a retrospective cohort study by using the national health insurance research database in Taiwan. Patients with a primary diagnosis of melanoma by the Taiwan Cancer Registry from 2009 to 2017 were recruited as the study population. The comparison group was never diagnosed with melanoma from 2000 to 2018. The Charlson comorbidity index was conducted to calculate the subjects' disease severity. The Cox proportional hazards model analysis was used to estimate the hazard ratio of death.

Results: We selected 476 patients, 55.5\% of whom had comorbidity. A higher prevalence of comorbidity was associated with a more advanced cancer stage. The mortality rate increased with an increasing level of comorbidity in both cohorts and was higher among melanoma patients. The interaction between melanoma and comorbidity resulted in an increased mortality rate.

Conclusion: An association between poorer survival and comorbidity was verified in this study. We found that the level of comorbidity was strongly associated with mortality. A higher risk of mortality was found in patients who had localized tumors, regional metastases, or distant metastases with more comorbidity scores. Advanced stage of melanoma patients with more comorbidities was significantly associated with the higher risk of mortality rate.

Keywords: melanoma, comorbidity, stage, mortality rate, survival, prognosis

\section{INTRODUCTION}

Cutaneous melanoma is now regarded as the fifth most common cancer in the United States. It was estimated that there were approximately 96,480 new cases, and 7,230 deaths due to melanoma of the skin will be newly diagnosed in 2019 (1). Despite being the deadliest form of skin cancer with a high mortality rate, many melanoma patients are cured after surgical excision of their primary tumor at 
an early stage; however, some still relapse after surgical excision. It is locally invasive and frequently spread out to regional lymph nodes and remote organs, including lung, liver, bone, and brain (2). The most common risk factors for malignant melanoma include family history, multiple moles, ultraviolet radiation, fair skin, and immunosuppression $(3,4)$. The incidence rate of melanoma has been increasing by between 3\% and 7\% per year globally for Caucasians (5). The incidence rate (less than 1/ $100,000)$ was lower in residents of Asia, including China, India, Singapore, and Japan (6). In Taiwan, the age-adjusted rate in 2006 for aggressive melanoma was $0.65 / 100,000(0.71 / 100,000$ for men, and 0.58/100,000 for women). The age distribution plot showed a peak among melanoma patients aged 70-79 years. According to the investigation of the incidence of melanoma from 1997 to 2008 by year and sex, the yearly incidence ranged from 0.66 to 1.24 (mean: 0.9 ) cases per 100,000 people in Taiwan (7). Excessive exposure to ultraviolet radiation is not the risk factor for most Taiwanese melanoma cases. In addition, 58\% of cutaneous melanoma belongs to acral lentiginous melanoma. Advanced disease is found in $50 \%$ of cases (8).

Studies on various prognostic factors affecting melanoma survival have been reported frequently. Age has been proven to be a very strong and independent predictor of survival outcome after accounting for all the dominant prognostic factors (9). Sentinel node biopsy is an important prognostic factor in melanoma (10). Lower socioeconomic status (SES) was associated with a decreased median survival time in a statistically significant amount for all stages of melanoma (11). Men have a greater risk of having advanced disease with a poorer outcome (12). Studies have shown that the presence of a melanoma on an axial site conferred a worse prognosis than an extremity site (9). Older age and advanced stage have significant negative effects on the survival of mucosal melanoma (13). The mitotic rate is a continuous prognostic variable. It is a strong independent predictor of outcome and should be assessed and recorded in all primary melanomas including in both initial and excision biopsies (14). Level of invasion has prognostic significance in univariate analysis and remains an independent predictor of outcome in more contemporary analyses. Clinical parameters such as age, sex, skin color, pigmentation status of the tumor, and site of the primary tumor play an important role for the outcome of patients. Ulceration is an adverse prognostic factor of melanoma (15). Stage and anatomic site, but not thickness (i.e., Breslow depth), race, or ethnicity, determine prognosis of mucosal melanomas (16). Stage, male gender, and age are associated with overall survival, along with SES and the presence of multiple comorbidities (17-19). Taken together, various prognostic factors affecting melanoma survival interact mostly synergistically. The prognostic influence of comorbidity and stage on melanoma has also caused many scientists to make many valuable reports. The prevalence of chronic disease in patients with melanoma varies from $19 \%$ to $80 \%$ (20). The presence of chronic conditions prevents physicians from aggressive treatment for melanoma patients, thereby increasing the mortality rate (20). The prevalence of multiple chronic conditions increased with age (21). The majority of studies from a systematic review reported decreased chemotherapy use (75\%) and inferior survival (69\%) for patients with comorbidities compared to those without comorbidities (22). Patient comorbidity has a substantial effect on the cancer stage at diagnosis (23). In a cohort study conducted using Danish registry data of 23,476 melanoma patients, Grann et al. (20) reported a higher prevalence of comorbidity associated with more advanced cancer stages. Similar results were acquired by Gonzalez et al. (24) using data from the Florida State Tumor Registry $(\mathrm{N}=32,074)$ in 1994 on colorectal, melanoma, breast, and prostate cancers. Comorbidity was associated with late-stage diagnosis in all four cancers, with the odds of $62 \%$ for late-stage melanoma (24). In Taiwan, just a few reports paid attention to malignant melanoma (25-27). Our current study aims at clarifying how comorbidity and stage may affect the prognosis of Taiwan melanoma patients from 2009 to 2017.

\section{MATERIALS AND METHODS}

\section{Data Source}

This study used the data from the National Health Insurance Research Database (NHIRD) and Taiwan Cancer Registry (TCR) that was constructed by the Health and Welfare Data Science Center (HWDSC). The NHIRD covered more than $99.99 \%$ of Taiwan's population of 23 million people in Taiwan. Two million beneficiaries were randomly sampled from the NHIRD in 2000. To protect patients' privacy, according to the "Personal Information Protection Act" and "Non-disclosure agreement of NHIRD," the original identification numbers were not disclosed. Data benefits include disease diagnosis of outpatient, inpatient, emergency medical claims, and cancer staging. The longitudinal characteristic of NHIRD allows researchers to identify a cohort based upon diagnoses and drug utilization, to trace the medical history, and to disclose clinical outcomes and related complications (28). TCR (29) is a nationwide population-based cancer registry system that provides detailed information on all cancer patients in Taiwan. Hospitals with more than 50-bed capacity that could supply outpatient and hospitalized cancer care are required to join in informing all newly diagnosed malignant neoplasms to the registry. In order to measure cancer care methods and treatment outcomes in Taiwan, the TCR constructed a long-form database with cancer staging and detailed treatment and recurrence information. Moreover, the long-form database included detailed information regarding cancer site-specific factors, such as laboratory values, tumor markers, and other clinical data related to patient care (30). The study had been approved by the ethical review board of the Chung Shan Medical University Hospital (CS1-20201) in Taiwan.

\section{Study Design and Outcome}

This study used a retrospective cohort study design. The study population was the primary site of melanoma (ICD-O-3=C44) from 2009 to 2017 in the TCR. The index date was admitted with 
the first diagnosis date of melanoma. The comparison group was defined as never diagnosis of melanoma (ICD-9-CM=172, ICD$10-\mathrm{CM}=\mathrm{C} 439$ ) from 2000 to 2018 (Figure 1). Due to the consistent index date between the melanoma group and the non-melanoma group, a 1:10 age and sex matching was conducted. The outcome variable was all-cause mortality. Both groups were followed up until the onset of death, or December 31, 2018, whichever occurred first.

The demographic variables included age, sex, monthly income, and residential region. We classified diagnoses of chronic diseases into 17 categories based on a modified version of the Charlson comorbidity index (CCI) (31). The 1 score of weight included myocardial infarction, congestive heart failure, peripheral vascular disease, cerebrovascular disease, dementia, chronic pulmonary disease, connective tissue disease, peptic ulcer disease, mild liver disease, and diabetes without chronic complication. The 2 scores of weight included diabetes with chronic complication, hemiplegia or paraplegia, and renal disease. The 3 scores of weight included any malignancy, including lymphoma and leukemia, except malignant neoplasm of skin, and severe liver disease. The 6 scores of weight included metastatic solid tumor and AIDS/HIV (Supplementary Table 1). The higher CCI-weighted total score represented the higher severity of the disease. The baseline characteristics also included age, sex, hypertension (ICD-9-CM=401-405, ICD-10-CM=I10-I15), and hyperlipidemia (ICD-9-CM=272, ICD-10-CM=E78). Those comorbidities were defined 1 year before the index date and at least two outpatient visits or once hospitalization.

\section{Statistical Analyses}

The comparison of continuous and categorical variables was done using Student's t-test, chi-square test, or Fisher's exact test as appropriate between melanoma and non-melanoma groups. Kaplan-Meier analysis was conducted to calculate the all-cause mortality among the two groups. The log-rank test was used to test the significance. The multivariate Cox proportional hazards model was used to estimate the hazard ratios (HRs) of death.
We used a time-dependent variable to assess the proportional hazards assumption. The $\mathrm{p}$ value was 0.2805 that it did not violate the proportional hazards assumption. For the unmeasured confounding factor, we performed E-value to define the minimum strength of the association for an unmeasured confounding effect between melanoma and nonmelanoma groups $(32,33)$. The statistical software was SAS version 9.4 (SAS Institute Inc., NC, USA).

\section{RESULTS}

\section{Study Population}

We identified 476 melanoma patients and 4,760 members of the matched comparison cohort. The majority were men $(56.7 \%)$, and more than half (85\%) were older than 55 years. According to tumor stage, $52(10.9 \%)$ in situ, $398(83.6 \%)$ had a localized tumor, $8(1.7 \%)$ had regional metastases, and $18(3.8 \%)$ had distant metastases (Table 1).

\section{Prevalence of Comorbidities}

Measurement of comorbidity resulted in 212 (44.5\%) melanoma patients with comorbidity score 0, 126 patients (26.5\%) with comorbidity score 1 , and $54(11.3 \%)$ with comorbidity score 2 . In the more severe comorbidity categories, 45 (9.5\%) had a comorbidity score 3 , and $39(8.2 \%)$ had a comorbidity score $\geq 4$. Diabetes without chronic complication was the most prevalent comorbidity, diagnosed in 83 patients (17.4\%) (Table 1). Chronic pulmonary disease was diagnosed in 63 patients $(13.2 \%)$, followed by peptic ulcer disease (58 patients, $12.2 \%$ ). The majority of melanoma patients were diagnosed with the localized stage (Table 1).

\section{All-Cause Mortality}

The mortality rate of the melanoma group in the Kaplan-Meier plot was significantly higher than that in the non-melanoma group (Figure 2). The mortality rate increased with increasing

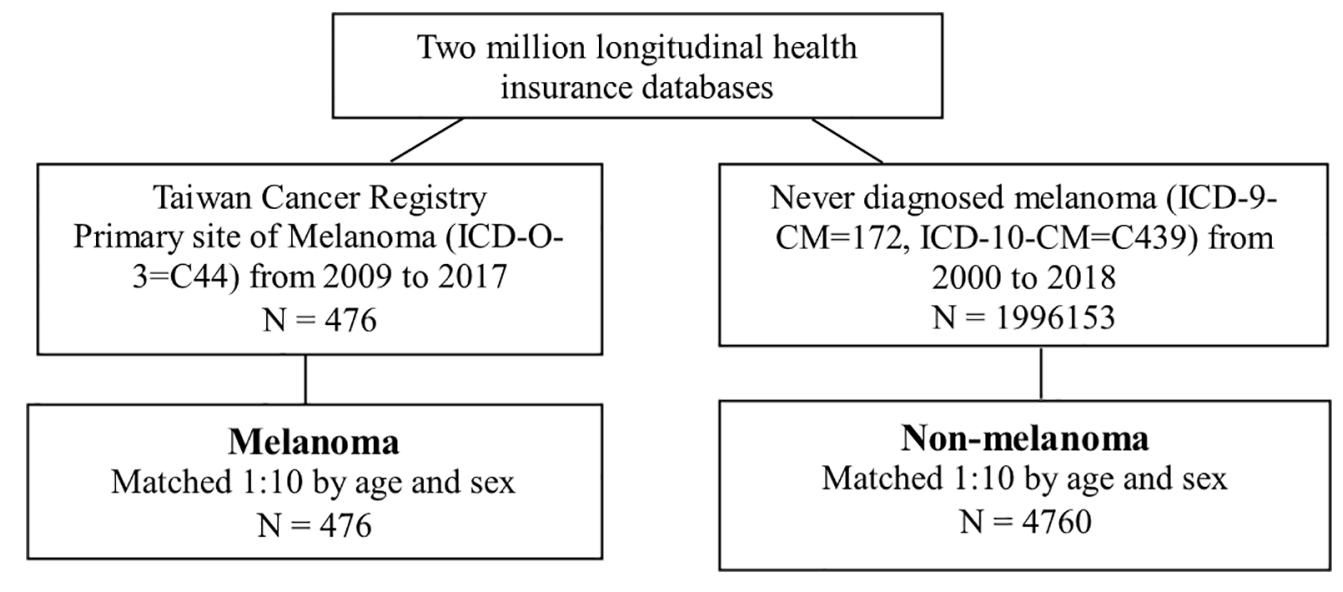

FIGURE 1 | Identified Melanoma and Non-melanoma groups. 
TABLE 1 | Demographic characteristics of melanoma and non-melanoma.

\begin{tabular}{|c|c|c|c|c|c|}
\hline & \multicolumn{2}{|c|}{ Non-melanoma $(\mathrm{N}=4,760)$} & \multicolumn{2}{|c|}{ Melanoma $(\mathrm{N}=476)$} & \multirow[t]{2}{*}{ p value } \\
\hline & $\mathbf{n}$ & $\%$ & $\mathbf{n}$ & $\%$ & \\
\hline Age & & & & & 1 \\
\hline$\leq 54$ & 710 & 14.9 & 71 & 14.9 & \\
\hline $55-69$ & 1,430 & 30.0 & 143 & 30.0 & \\
\hline$\geq 70$ & 2,620 & 55.1 & 262 & 55.1 & \\
\hline Mean \pm SD & \multicolumn{2}{|c|}{$69.91 \pm 15.66$} & \multicolumn{2}{|c|}{$69.91 \pm 15.67$} & 1 \\
\hline Sex & & & & & 1 \\
\hline Female & 2,060 & 43.3 & 206 & 43.3 & \\
\hline Male & 2,700 & 56.7 & 270 & 56.7 & \\
\hline Monthly income (NT\$) & & & & & 0.5469 \\
\hline$<20,000$ & 1,527 & 32.1 & 144 & 30.3 & \\
\hline $20,001-40,000$ & 2,349 & 49.3 & 235 & 49.4 & \\
\hline$>40,000$ & 884 & 18.6 & 97 & 20.4 & \\
\hline Residential region & & & & & 0.0014 \\
\hline Northern & 1,989 & 41.8 & 242 & 50.8 & \\
\hline Central & 1,116 & 23.4 & 87 & 18.3 & \\
\hline Southern & 1,212 & 25.5 & 111 & 23.3 & \\
\hline Others & 443 & 9.3 & 36 & 7.6 & \\
\hline \multicolumn{6}{|l|}{ Stage of melanoma } \\
\hline 0 & & & 52 & 10.9 & \\
\hline I & & & 292 & 61.3 & \\
\hline$\|$ & & & 106 & 22.3 & \\
\hline III & & & 8 & 1.7 & \\
\hline IV & & & 18 & 3.8 & \\
\hline Hypertension & 1,997 & 42.0 & 242 & 50.8 & 0.0002 \\
\hline Hyperlipidemia & 986 & 20.7 & 108 & 22.7 & 0.3123 \\
\hline \multicolumn{6}{|c|}{ Categories of Charlson comorbidity index score } \\
\hline Myocardial infarction & 44 & 0.9 & 5 & 1.1 & 0.7854 \\
\hline Congestive heart failure & 197 & 4.1 & 14 & 2.9 & 0.205 \\
\hline Peripheral vascular disease & 56 & 1.2 & 8 & 1.7 & 0.3398 \\
\hline Cerebrovascular & 444 & 9.3 & 56 & 11.8 & 0.0845 \\
\hline Dementia & 175 & 3.7 & 18 & 3.8 & 0.9077 \\
\hline Chronic pulmonary disease & 484 & 10.2 & 63 & 13.2 & 0.0370 \\
\hline Connective tissue disease & 26 & 0.5 & 4 & 0.8 & 0.4176 \\
\hline Peptic ulcer disease & 473 & 9.9 & 58 & 12.2 & 0.1214 \\
\hline Mild liver disease & 187 & 3.9 & 33 & 6.9 & 0.0018 \\
\hline Diabetes without chronic complication & 820 & 17.2 & 83 & 17.4 & 0.9079 \\
\hline Diabetes with chronic complication & 244 & 5.1 & 26 & 5.5 & 0.7519 \\
\hline Hemiplegia or paraplegia & 19 & 0.4 & 3 & 0.6 & 0.44549 \\
\hline Renal disease & 310 & 6.5 & 41 & 8.6 & 0.0806 \\
\hline Any malignancy & 50 & 1.1 & 21 & 4.4 & $<0.0001$ \\
\hline Charlson comorbidity index score & & & & & $<0.0001$ \\
\hline 0 & 2,663 & 55.95 & 212 & 44.5 & \\
\hline 1 & 1,024 & 21.51 & 126 & 26.5 & \\
\hline 2 & 478 & 10.04 & 54 & 11.3 & \\
\hline 3 & 280 & 5.88 & 45 & 9.5 & \\
\hline$\geq 4$ & 315 & 6.62 & 39 & 8.2 & \\
\hline
\end{tabular}

IFisher's exact test.

comorbidity in both cohorts and was higher among melanoma patients (Figure 3). The mortality rate for melanoma patients with comorbid score 1 was 57.42 per 1,000 person-years after diagnosis compared with 50.50 in the comparison cohort. In the most severe comorbidity group $(\geq 4)$, the mortality rate was 147.21 per 1,000 person-years in the melanoma cohort compared with 149.43 in the comparison cohort. The mortality rate ratio (MRR) was 1.59 (95\% CI: 1.28-1.98). The number of melanoma patients at stages 0 , I, II, III, and IV was $52(10.9 \%)$, $292(61.3 \%), 106(22.3 \%), 8(1.7 \%)$, and $18(3.8 \%)$, respectively (Table 1). In the univariate analysis, old age was associated with a significantly increased risk of death $(p<0.0001)$. Patients greater than and equal to 70 years of age ( $\mathrm{HR}=18.64,95 \% \mathrm{CI}$ : 10.96-31.72) had a worse prognosis than patients less than and equal to 54 years of age. The risk of death for people with comorbidity scores $1,2,3$, and $\geq 4$ was $2.65,4.88,5.48$, and 7.77 , respectively (Table 2). Table 3 demonstrated the subgroup analysis of the risk of death between melanoma and nonmelanoma groups. The risk of death for patients aged $\leq 54$ between melanoma and non-melanoma group was the highest $(\mathrm{HR}=13.08,95 \% \mathrm{CI}=4.41-38.77, \mathrm{p}<0.0001)$ among the three age groups. For patients between melanoma and non-melanoma 


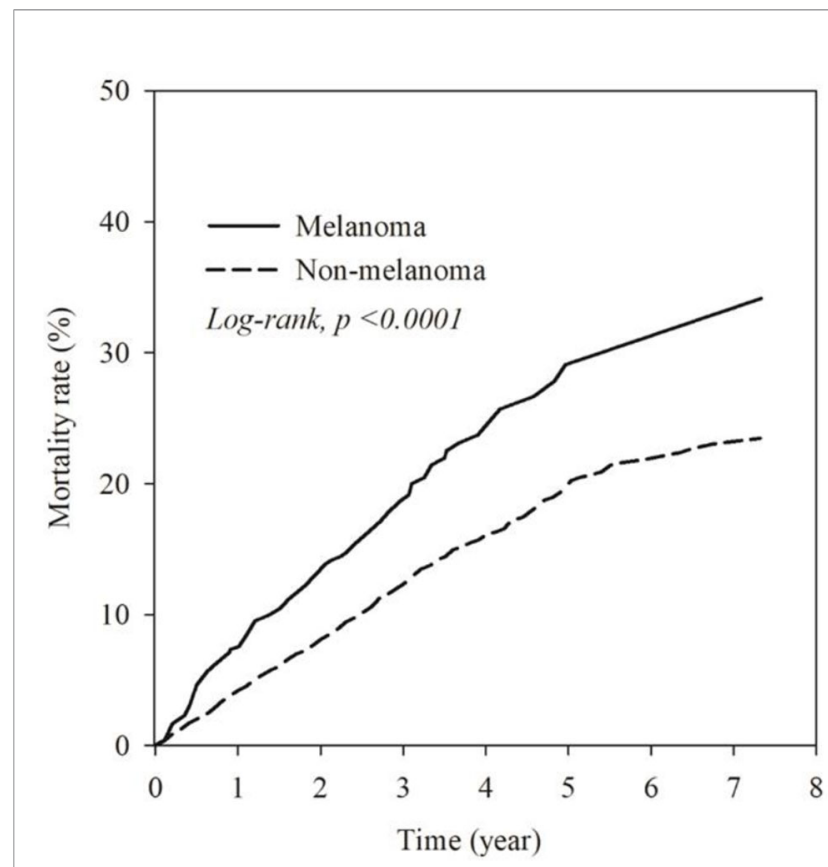

FIGURE 2 | Kaplan-Meier plot for the mortality rate in melanoma group and non-melanoma group.

groups with comorbidity score 0 , the HR risk of death was 2.40 (95\% CI: $1.56-3.68, \mathrm{p}<0.0001$ ). Table 4 demonstrated the higher risk of mortality for people with comorbidity score 3 $(\mathrm{HR}=2.79,95 \% \mathrm{CI}: 1.46-5.30, \mathrm{p}=0.002)$ and score $\geq 4(\mathrm{HR}=$ 2.75, 95\% CI: 1.39-5.44, $\mathrm{p}=0.004)$. Increased comorbidity was related to the advanced cancer stage at diagnosis. The HR risk of mortality in melanoma patients with comorbidity scores $1, \geq 2$ at stages 0 and I was 2.61 (95\% CI: 1.01-6.78) and 2.62 (95\% CI: 1.12-6.13), respectively. For patients with comorbidity scores $1, \geq 2$ at stage II, the HR of risk of mortality was 3.26 (95\% CI: 1.20-8.84). The E-values for patients with comorbidity scores 1 , $\geq 2$ at stages 0 and I and comorbidity score $\geq 2$ at stage II were $4.66,4.68$, and 5.97, respectively. We found in this study that the advanced stage of melanoma patients with more comorbidities was significantly associated with the higher risk of mortality rate.

\section{DISCUSSION}

In this nationwide registry-based cohort study, we included 476 men and women diagnosed with melanoma. According to tumor stage, 52 (10.9\%) in situ, 398 (83.6\%) had a localized tumor, 8 (1.7\%) had regional metastases, 18 (3.8\%) had distant metastases, and 364 (55.5\%) suffered from one or more comorbidities (Table 1). Our study unraveled an association between a higher prevalence of comorbidities and a higher risk of death. The more comorbidity, the higher the risk of mortality. Comorbidity commonly is associated with poorer survival. Old age was associated with a significantly increased risk of death. In our study, patients greater than and equal to 70 years of age ( $\mathrm{HR}=18.64,95 \% \mathrm{CI}: 10.96-31.72)$ had a worse risk of death than patients 55-69 years of age (Table 2). There was an association between older age and more advanced tumors at diagnosis, leading to higher mortality among elderly people $(34,35)$. In Taiwan, malignant melanoma is an uncommon but fatal disease. One possible reason for low survival was that farmers delayed the diagnosis to old age. The comorbidities, or neglecting consciousness of melanoma, adverse effects of their treatment, or disguising the symptoms of the disease by the patient or doctor could be another possibility for late detection in people with comorbid conditions (36).

Gonzalez et al. (24) demonstrated that having comorbidity and cancer (colorectal, melanoma, breast, prostate) has resulted in late diagnosis of cancer. A previous study from Grann et al. (20) showed that comorbidity initiated a higher risk of complications and worse functional status, decreased quality of life, and poorer survival-especially in older patients. Houterman et al. (37) reported that increased levels of severe comorbidity led to less aggressive treatment that negatively influenced the survival of elderly patients aged 60-79 years. Comorbidities could worsen comorbid diseases and lower the functional status of metastatic melanoma patients receiving curative treatment (20). Research from Taiwan reported that a worse prognosis with great differences was mostly found in histologic subtypes, advanced stages, and acral lentiginous melanoma. Melanoma patients had a poorer prognosis when they were diagnosed with more advanced stage (26). Some studies suggested that coexistent disease was associated with

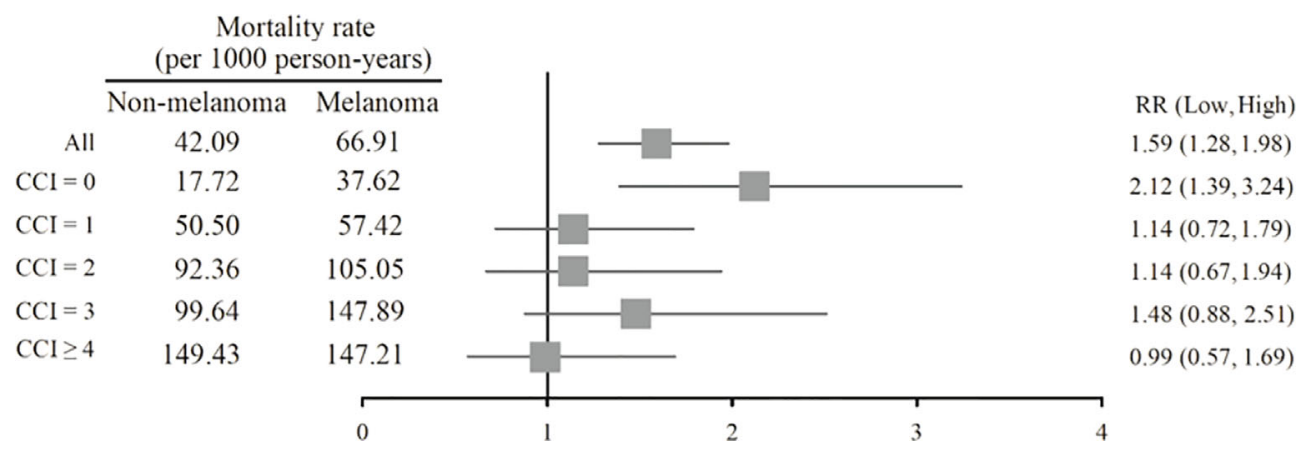

FIGURE 3 | Forest plot of mortality rate according to CCl subgroups. 
TABLE 2 | Cox proportional hazards model analysis for risk of death.

\begin{tabular}{|c|c|c|c|c|}
\hline & \multicolumn{2}{|c|}{ Univariate } & \multicolumn{2}{|c|}{ Multivariate $^{\dagger}$} \\
\hline & HR $(95 \%$ Cl) & $p$ value & HR (95\% Cl) & $p$ value \\
\hline \multicolumn{5}{|l|}{ Group } \\
\hline Non-melanoma & Reference & & Reference & \\
\hline Melanoma & $1.58(1.27-1.97)$ & $<0.0001$ & $1.46(1.17-1.82)$ & $<0.001$ \\
\hline \multicolumn{5}{|l|}{ Age } \\
\hline$\leq 54$ & Reference & & Reference & \\
\hline $55-69$ & 2.67 (1.49-4.77) & 0.001 & 2.21 (1.23-3.96) & 0.008 \\
\hline$\geq 70$ & 18.64 (10.96-31.72) & $<0.0001$ & $11.76(6.84-20.24)$ & $<0.0001$ \\
\hline \multicolumn{5}{|l|}{ Sex } \\
\hline Female & Reference & & Reference & \\
\hline Male & 1.15 (0.99-1.33) & 0.078 & $1.10(0.94-1.28)$ & 0.246 \\
\hline \multicolumn{5}{|c|}{ Monthly income (NT\$) } \\
\hline$<20,000$ & Reference & & Reference & \\
\hline $20,001-40,000$ & $0.90(0.77-1.05)$ & 0.186 & 1.09 (0.86-1.39) & 0.473 \\
\hline$>40,000$ & $0.58(0.46-0.74)$ & $<0.0001$ & $1.11(0.88-1.42)$ & 0.380 \\
\hline \multicolumn{5}{|l|}{ Residential region } \\
\hline Northern & Reference & & Reference & \\
\hline Central & $1.26(1.04-1.51)$ & 0.016 & 1.07 (0.88-1.30) & 0.503 \\
\hline Southern & 1.19 (0.99-1.43) & 0.061 & 1.07 (0.89-1.29) & 0.487 \\
\hline Others & $0.90(0.67-1.20)$ & 0.471 & $0.81(0.60-1.09)$ & 0.158 \\
\hline Hypertension & $2.22(1.91-2.58)$ & $<0.0001$ & 1.09 (0.93-1.28) & 0.304 \\
\hline Hyperlipidemia & $0.66(0.54-0.82)$ & $<0.001$ & $0.43(0.35-0.54)$ & $<0.0001$ \\
\hline \multicolumn{5}{|c|}{ Charlson comorbidity index score } \\
\hline 0 & Reference & & Reference & \\
\hline 1 & $2.65(2.15-3.26)$ & $<0.0001$ & $1.99(1.61-2.47)$ & $<0.0001$ \\
\hline 2 & $4.88(3.92-6.09)$ & $<0.0001$ & $3.13(2.49-3.93)$ & $<0.0001$ \\
\hline 3 & $5.48(4.26-7.04)$ & $<0.0001$ & $3.36(2.59-4.35)$ & $<0.0001$ \\
\hline$\geq 4$ & 7.77 (6.19-9.75) & $<0.0001$ & $4.77(3.75-6.06)$ & $<0.0001$ \\
\hline
\end{tabular}

${ }^{+}$Adjusted for age, sex, hypertension, hyperlipidemia, monthly income, residential region, and Charlson comorbidity index score.

worse survival and increased the possibility of being diagnosed with distant metastasis $(8,23,38-43)$. Our study revealed the association between comorbidity and stage that may influence the prognosis of melanoma patients in Taiwan. Table 4 showed that for people with comorbidity scores 3 and $\geq 4$, HR of the risk of mortality was relatively higher than scores 1 and 2 . The risk of mortality for patients with comorbidity score $\geq 2$ at stages 0 , I was a little higher than those with comorbidity score 1 . The risk of mortality for patients with comorbidity score $\geq 2$ at stage II was significantly higher than those with comorbidity score 1 . Though the risk of mortality for patients with comorbidity score $\geq 2$ at stages III, IV was lower than those with comorbidity score 1 , no major differences were found because of too small a patient population.

Comorbidity might reduce survival because curative treatment is used less frequently in older patients. Consequently, survival of patients older than 70 years was not significantly influenced by comorbidity (44). Bradley et al. (45) reported that men with more comorbid conditions were less likely to receive treatment than those without comorbidities. The decision to receive treatment was determined mainly by the patient's age, disease stage, tumor characteristics, and experience of the urologist $(44,46)$. Fowler et al. (47) revealed that associations between age and comorbidity were highly significant ( $p<0.0001)$, as the age-adjusted risk of comorbid death was 5.7 times greater in men with severe compared to those without comorbidities. Two previous studies showed that less aggressive treatment for melanoma among patients with comorbidity may affect the mortality rate because of some of the interactions between melanoma and comorbidity. Decreased function of the immune system in older patients with more comorbidities may result in higher mortality because of the interaction between comorbidity and melanoma $(48,49)$. Koppie et al. (50) also reported that the conditions required for treatment, such as a history of comorbidities, age, performance status, and other related factors, are important for melanoma patients when they plan to receive necessary treatment. Generally, elderly patients with more comorbidities are less likely to receive the most aggressive chemotherapy combinations for avoiding a high risk of significant morbidity (50).

The minority of melanoma patients (44.5\%) had none of the selected comorbidities since their melanoma was diagnosed. In the remaining $55.5 \%$ of the melanoma cohort with some prevalent comorbidity, the most common comorbid diseases were diabetes without chronic complication, chronic pulmonary disease, peptic ulcer disease, and cerebrovascular (Table 1). Among melanoma and non-melanoma groups, the proportion diagnosed with severe comorbidity increased with an increasing mortality rate, and the level of comorbidity was strongly associated with mortality (Figure 3).

However, the weight of the association differs by specific comorbid disease, patient age, cancer characteristics, and overall comorbidity burden $(23,34,35)$. Comorbidity can be measured by counting the number of coexisting illnesses diagnosed in a cancer patient or by using a comorbidity index that integrates the 
TABLE 3 | Subgroup analysis of risk of death between melanoma and non-melanoma group.

\begin{tabular}{|c|c|c|c|c|c|c|}
\hline & \multicolumn{2}{|c|}{ Non-melanoma } & \multicolumn{2}{|c|}{ Melanoma } & \multirow[t]{2}{*}{$\mathrm{HR}^{\dagger}(95 \% \mathrm{Cl})$} & \multirow[t]{2}{*}{ p value } \\
\hline & $\mathbf{N}$ & No. of death & $\mathbf{N}$ & No. of death & & \\
\hline \multicolumn{7}{|l|}{ Age } \\
\hline$\leq 54$ & 710 & 7 & 71 & 7 & $13.08(4.41-38.77)$ & $<0.0001$ \\
\hline $55-69$ & 1,430 & 47 & 143 & 14 & $2.85(1.55-5.23)$ & $<0.001$ \\
\hline$\geq 70$ & 2,620 & 571 & 262 & 72 & $\begin{array}{l}1.33(1.04-1.70) \\
p \text { for interaction }\end{array}$ & $\begin{array}{c}0.024 \\
<0.0001\end{array}$ \\
\hline \multicolumn{7}{|l|}{ Sex } \\
\hline Female & 2,060 & 235 & 206 & 39 & $1.63(1.16-2.29)$ & 0.005 \\
\hline Male & 2,700 & 390 & 270 & 54 & $\begin{array}{l}1.50(1.13-2.00) \\
p \text { for interaction }=\end{array}$ & $\begin{array}{c}0.006 \\
0.6331\end{array}$ \\
\hline \multicolumn{7}{|c|}{ Monthly income (NT\$) } \\
\hline$<20,000$ & 1,527 & 231 & 144 & 34 & $1.76(1.22-2.54)$ & 0.003 \\
\hline $20,001-40,000$ & 2,349 & 320 & 235 & 44 & $1.33(0.97-1.83)$ & 0.078 \\
\hline$>40,000$ & 884 & 74 & 97 & 15 & $\begin{array}{l}1.93(1.09-3.42) \\
\text { p for interaction }=\end{array}$ & $\begin{array}{c}0.024 \\
0.3457\end{array}$ \\
\hline \multicolumn{7}{|l|}{ Residential region } \\
\hline Northern & 1,989 & 236 & 242 & 42 & 1.55 (1.12-2.16) & 0.009 \\
\hline Central & 1,116 & 172 & 87 & 17 & $1.29(0.78-2.13)$ & 0.322 \\
\hline Southern & 1,212 & 171 & 111 & 27 & $1.82(1.20-2.74)$ & 0.005 \\
\hline Others & 443 & 46 & 36 & 7 & $\begin{array}{c}1.37(0.61-3.09) \\
\text { p for interaction }=\end{array}$ & $\begin{array}{l}0.447 \\
0.775\end{array}$ \\
\hline \multicolumn{7}{|c|}{ Charlson comorbidity index score } \\
\hline 0 & 2,663 & 158 & 212 & 25 & $2.40(1.56-3.68)$ & $<0.0001$ \\
\hline 1 & 1,024 & 156 & 126 & 21 & $1.22(0.77-1.93)$ & 0.394 \\
\hline 2 & 478 & 124 & 54 & 15 & $1.01(0.58-1.76)$ & 0.977 \\
\hline 3 & 280 & 75 & 45 & 17 & $1.67(0.98-2.86)$ & 0.060 \\
\hline$\geq 4$ & 315 & 112 & 39 & 15 & $\begin{array}{l}1.20(0.70-2.08) \\
p \text { for interaction }=\end{array}$ & $\begin{array}{c}0.509 \\
0.0799\end{array}$ \\
\hline \multicolumn{7}{|c|}{ Charlson comorbidity index score } \\
\hline 0 & 2,663 & 158 & 212 & 25 & $2.40(1.56-3.68)$ & $<0.0001$ \\
\hline 1 & 1,024 & 156 & 126 & 21 & $1.22(0.77-1.93)$ & 0.394 \\
\hline$\geq 2$ & 1,073 & 311 & 138 & 47 & $\begin{array}{l}1.25(0.92-1.71) \\
\text { p for interaction }=\end{array}$ & $\begin{array}{l}0.152 \\
0.029\end{array}$ \\
\hline
\end{tabular}

${ }^{+}$Adjusted for age, sex, monthly income, residential region, hypertension, and hyperlipidemia.

TABLE 4 | Cox proportional hazards model analysis for risk of mortality in melanoma patients.

\begin{tabular}{|c|c|c|c|c|}
\hline & $\mathbf{N}$ & Numbers of death & $\mathrm{HR}^{\dagger}(95 \% \mathrm{Cl})$ & $p$ value \\
\hline \multicolumn{5}{|c|}{ Charlson comorbidity index score } \\
\hline 0 & 212 & 25 & Reference & \\
\hline 1 & 126 & 21 & $1.24(0.69-2.23)$ & 0.475 \\
\hline 2 & 54 & 15 & $1.58(0.81-3.11)$ & 0.184 \\
\hline 3 & 45 & 17 & $2.79(1.46-5.30)$ & 0.002 \\
\hline$\geq 4$ & 39 & 15 & $2.75(1.39-5.44)$ & 0.004 \\
\hline \multicolumn{5}{|c|}{ Stage 0,1} \\
\hline \multicolumn{5}{|c|}{ Charlson comorbidity index score } \\
\hline 0 & 155 & 8 & Reference & \\
\hline 1 & 88 & 11 & $2.61(1.01-6.78)$ & 0.048 \\
\hline$\geq 2$ & 101 & 23 & $2.62(1.12-6.13)$ & 0.026 \\
\hline \multicolumn{5}{|c|}{ Stage II } \\
\hline \multicolumn{5}{|c|}{ Charlson comorbidity index score } \\
\hline 0 & 45 & 12 & Reference & \\
\hline 1 & NA & NA & $0.44(0.14-1.33)$ & 0.144 \\
\hline$\geq 2$ & NA & NA & $3.26(1.20-8.84)$ & 0.020 \\
\hline \multicolumn{5}{|c|}{ Stage III, IV } \\
\hline \multicolumn{5}{|c|}{ Charlson comorbidity index score } \\
\hline 0 & 12 & 5 & Reference & \\
\hline 1 & NA & NA & $3.86(0.59-25.22)$ & 0.158 \\
\hline$\geq 2$ & NA & NA & 5.17 (0.69-38.92) & 0.110 \\
\hline
\end{tabular}

${ }^{\dagger}$ Adjusted for age, sex, monthly income, residential region, hypertension, and hyperlipidemia. NA, not available. 
number and severity of the comorbidity (51). Nowadays, the CCI is the most commonly applied index for comorbidities in cancer patients. The CCI score is the sum of weights of a patient's coexisting conditions based on 19 disease categories. The weights originated from relative risk assessment acquired from a regression model. They are usually assigned from 1 to 6 points and then collapsed into categories of 0 point, 1 to 2 points, 3 to 4 points, and 5 or more points, respectively. The CCI has been previously verified as a prognostic method of comorbidity for some index cancers (31).

A study previously showed that both the survival and treatment of patients were affected by age and the extent of comorbidity. Racial differences in survival were greatest for patients without comorbidities and less pronounced at higher levels of comorbidity. Comorbidity elicited differential impact for prognosis, treatment, and survival (52). However, the outcomes of this study by using the CCI score were similar with many reports in Western countries $(24,39)$. Comorbidities that influence prognosis seriously may differ between Eastern and Western countries. We expect that future research can focus on which of these comorbidities may most seriously affect the prognosis of melanoma patients.

There is a limitation in this study. From Taiwan's NHIRD, we selected random samples of 2 million from 23 million beneficiaries. Because melanoma is an unusual illness in Taiwan, the sample size of melanoma patients was relatively small in our research. However, NHIRD is a randomly selected representative sample of Taiwan's general population. This may avoid sampling deviation or selection bias and provide more nationwide information in this study.

In conclusion, melanoma patients in Taiwan with comorbidity were associated with poorer survival. The level of comorbidity was robustly associated with the mortality rate. The presence of severe comorbidity was associated with an advanced stage of melanoma. The mortality rate was higher among patients with more comorbidities, and the influence of comorbidity varied by stage. Old age was associated with a significantly increased risk of death. A higher risk of mortality

\section{REFERENCES}

1. Siegel RL, Miller KD, Jemal A. Cancer Statistics, 2019. CA Cancer J Clin (2019) 69:7-34. doi: 10.3322/caac.21551

2. Damsky WE, Theodosakis N, Bosenberg M. Melanoma Metastasis: New Concepts and Evolving Paradigms. Oncogene (2014) 33:2413-22. doi: 10.1038/onc.2013.194

3. Lo JA, Fisher DE. The Melanoma Revolution: From UV Carcinogenesis to a New Era in Therapeutics. Science (2014) 346:945-9. doi: 10.1126/ science. 1253735

4. Eggermont AM, Spatz A, Robert C. Cutaneous Melanoma. Lancet (2014) 383:816-27. doi: 10.1016/S0140-6736(13)60802-8

5. Erdei E, Torres SM. A New Understanding in the Epidemiology of Melanoma. Expert Rev Anticancer Ther (2010) 10:1811-23. doi: 10.1586/era.10.170

6. Shoo BA, Kashani-Sabet M. Melanoma Arising in African-, Asian-, Latino- and Native-American Populations. Semin Cutan Med Surg (2009) 28:96-102. doi: 10.1016/j.sder.2009.04.005

7. Hwang CY, Chen YJ, Lin MW, Chen TJ, Chu SY, Chen CC, et al. Elevated Risk of Second Primary Cancer in Patients With Cutaneous Malignant Melanoma: was found in patients who had localized tumors, regional metastases, or distant metastases with more comorbidity scores. Our study demonstrated that comorbidity and stage had an impact on the prognosis of Taiwan melanoma patients.

\section{DATA AVAILABILITY STATEMENT}

The data in this study we used were from the National Health Insurance Research Database and Taiwan Cancer Registry.

\section{AUTHOR CONTRIBUTIONS}

All authors listed have contributed to the work and approved its publication. Contributors: All other authors (YSH, PNC, SCC, JYH, YHW and JCCW) provided their input by contributing to the conceptualization. CKC contributed to the editing of the article.

\section{ACKNOWLEDGMENTS}

I would like to express my thanks to Professor James ChengChung Wei, Professor Yih-Shou Hsieh, Professor Pei-Ni Chen, Professor Shu-Chen Chu, and Dr. Jing-Yang Huang, who all served as scientific advisers, and Yu-Hsun Wang, who collected data for this study. The authors also wish to thank all colleagues from the Department of Medical Research, Chung Shan Medical University Hospital, Taichung, Taiwan.

\section{SUPPLEMENTARY MATERIAL}

The Supplementary Material for this article can be found online at: https://www.frontiersin.org/articles/10.3389/fonc.2022.846760/ full\#supplementary-material
A Nationwide Cohort Study in Taiwan. J Dermatol Sci (2010) 60:167-72. doi: 10.1016/j.jdermsci.2010.10.004

8. Chang JW. Cutaneous Melanoma: Taiwan Experience and Literature Review. Chang Gung Med J (2010) 33:602-12.

9. Balch CM, Soong SJ, Gershenwald JE, Thompson JF, Coit DG, Atkins MB et al. Age as a Prognostic Factor in Patients With Localized Melanoma and Regional Metastases. Ann Surg Oncol (2013) 20:3961-8. doi: 10.1245/s10434013-3100-9

10. Wu PC, Chen YC, Chen HM, Chen LW. Prognostic Factors and PopulationBased Analysis of Melanoma With Sentinel Lymph Node Biopsy. Sci Rep (2021) 11:20524. doi: 10.1038/s41598-021-99950-1

11. Salvaggio C, Han SW, Martires K, Robinson E, Madankumar R, Gumaste P, et al. Impact of Socioeconomic Status and Ethnicity on Melanoma Presentation and Recurrence in Caucasian Patients. Oncology (2016) 90:79-87. doi: 10.1159/000441524

12. Clark WH Jr, Elder DE, Guerry DT, Braitman LE, Trock BJ, Schultz D, et al. Model Predicting Survival in Stage I Melanoma Based on Tumor Progression. J Natl Cancer Inst (1989) 81:1893-904. doi: 10.1093/jnci/81.24.1893

13. Sarac E, Amaral T, Keim U, Leiter U, Forschner A, Eigentler TK, et al. Prognostic Factors in 161 Patients With Mucosal Melanoma: A Study of 
German Central Malignant Melanoma Registry. J Eur Acad Dermatol Venereol (2020) 34:2021-5. doi: 10.1111/jdv.16306

14. Amin MB, Greene FL, Edge SB, Compton CC, Gershenwald JE, Brookland RK, et al. The Eighth Edition AJCC Cancer Staging Manual: Continuing to Build a Bridge From a Population-Based to a More "Personalized" Approach to Cancer Staging. CA Cancer J Clin (2017) 67:93-9. doi: 10.3322/caac.21388

15. Bobos M. Histopathologic Classification and Prognostic Factors of Melanoma: A 2021 Update. Ital J Dermatol Venerol (2021) 156:300-21. doi: 10.23736/ S2784-8671.21.06958-3

16. Altieri L, Eguchi M, Peng DH, Cockburn M. Predictors of Mucosal Melanoma Survival in a Population-Based Setting. J Am Acad Dermatol (2019) 81:136142 e2. doi: 10.1016/j.jaad.2018.09.054

17. Feigelson HS, Powers JD, Kumar M, Carroll NM, Pathy A, Ritzwoller DP. Melanoma Incidence, Recurrence, and Mortality in an Integrated Healthcare System: A Retrospective Cohort Study. Cancer Med (2019) 8:4508-16. doi: 10.1002/cam4.2252

18. Cheng E, Soulos PR, Irwin ML, Cespedes Feliciano EM, Presley CJ, Fuchs CS, et al. Neighborhood and Individual Socioeconomic Disadvantage and Survival Among Patients With Nonmetastatic Common Cancers. JAMA Netw Open (2021) 4:e2139593. doi: 10.1001/jamanetworkopen.2021.39593

19. Syriopoulou E, Morris E, Finan PJ, Lambert PC, Rutherford MJ. Understanding the Impact of Socioeconomic Differences in Colorectal Cancer Survival: Potential Gain in Life-Years. Br J Cancer (2019) 120:10528. doi: 10.1038/s41416-019-0455-0

20. Grann AF, Froslev T, Olesen AB, Schmidt H, Lash TL. The Impact of Comorbidity and Stage on Prognosis of Danish Melanoma Patients, 19872009: A Registry-Based Cohort Study. Br J Cancer (2013) 109:265-71. doi: $10.1038 /$ bjc. 2013.246

21. Salive ME. Multimorbidity in Older Adults. Epidemiol Rev (2013) 35:75-83. doi: 10.1093/epirev/mxs009

22. Lee L, Cheung WY, Atkinson E, Krzyzanowska MK. Impact of Comorbidity on Chemotherapy Use and Outcomes in Solid Tumors: A Systematic Review. J Clin Oncol (2011) 29:106-17. doi: 10.1200/JCO.2010.31.3049

23. Gurney J, Sarfati D, Stanley J. The Impact of Patient Comorbidity on Cancer Stage at Diagnosis. Br J Cancer (2015) 113:1375-80. doi: 10.1038/bjc.2015.355

24. Gonzalez EC, Ferrante JM, Van Durme DJ, Pal N, Roetzheim RG. Comorbid Illness and the Early Detection of Cancer. South Med J (2001) 94:913-20. doi: 10.1097/00007611-200109000-00020

25. Chang WS, Yang KC, Chen BF. Malignant Melanoma Metastatic to the Stomach: Upper G-I Endoscopic Finding-Report of a Case. Zhonghua Yi Xue Za Zhi (Taipei) (1990) 45:280-3.

26. Chen YJ, Wu CY, Chen JT, Shen JL, Chen CC, Wang HC. Clinicopathologic Analysis of Malignant Melanoma in Taiwan. J Am Acad Dermatol (1999) 41:945-9. doi: 10.1016/S0190-9622(99)70251-3

27. Soong CY, Liu HN, Ger LP, Chu TL, Syu HL, Tseng HH. Malignant Melanoma: A Clinicopathologic Study of 22 Cases. J Formos Med Assoc (1991) 90:365-70.

28. Tai CS, Wu JF, Chen HL, Hsu HY, Chang MH, Ni YH. Modality of Treatment and Potential Outcome of Wilson Disease in Taiwan: A Population-Based Longitudinal Study. J Formos Med Assoc (2018) 117:421-6. doi: 10.1016/ j.jfma.2017.05.008

29. Chiang CJ, Wang YW, Lee WC. Taiwan's Nationwide Cancer Registry System of 40 Years: Past, Present, and Future. J Formos Med Assoc (2019) 118:856-8. doi: 10.1016/j.jma.2019.01.012

30. Chiang CJ, You SL, Chen CJ, Yang YW, Lo WC, Lai MS. Quality Assessment and Improvement of Nationwide Cancer Registration System in Taiwan: A Review. Jpn J Clin Oncol (2015) 45:291-6. doi: 10.1093/jjco/hyu211

31. Sundararajan V, Henderson T, Perry C, Muggivan A, Quan H, Ghali WA. New ICD-10 Version of the Charlson Comorbidity Index Predicted inHospital Mortality. J Clin Epidemiol (2004) 57:1288-94. doi: 10.1016/ j.jclinepi.2004.03.012

32. Haneuse S, VanderWeele TJ, Arterburn D. Using the E-Value to Assess the Potential Effect of Unmeasured Confounding in Observational Studies. JAMA (2019) 321:602-3. doi: 10.1001/jama.2018.21554

33. VanderWeele TJ,Ding P. Sensitivity Analysis in Observational Research: Introducing the E-Value. Ann Intern Med (2017) 167:268-74. doi: 10.7326/M16-2607

34. Lasithiotakis KG, Petrakis IE, Garbe C. Cutaneous Melanoma in the Elderly: Epidemiology, Prognosis and Treatment. Melanoma Res (2010) 20:163-70. doi: 10.1097/CMR.0b013e328335a8dd
35. Norgaard C, Glud M, Gniadecki R. Are All Melanomas Dangerous? Acta Derm Venereol (2011) 91:499-503. doi: 10.2340/00015555-1177

36. Chang HY, Feng HL, Wang L, Chou P, Wang PF, Incidence T. Prevalence, and Survival of Malignant Melanoma in Taiwan. Value Health (2014) 17: A740. doi: 10.1016/j.jval.2014.08.135

37. Houterman S, Janssen-Heijnen ML, Hendrikx AJ, van den Berg HA, Coebergh JW. Impact of Comorbidity on Treatment and Prognosis of Prostate Cancer Patients: A Population-Based Study. Crit Rev Oncol Hematol (2006) 58:60-7. doi: 10.1016/j.critrevonc.2005.08.003

38. Louwman WJ, Janssen-Heijnen ML, Houterman S, Voogd AC, van der Sangen MJ, Nieuwenhuijzen GA, et al. Less Extensive Treatment and Inferior Prognosis for Breast Cancer Patient With Comorbidity: A Population-Based Study. Eur J Cancer (2005) 41:779-85. doi: 10.1016/j.ejca.2004.12.025

39. Cronin-Fenton DP, Norgaard M, Jacobsen J, Garne JP, Ewertz M, Lash TL, et al. Comorbidity and Survival of Danish Breast Cancer Patients From 1995 to 2005. Br J Cancer (2007) 96:1462-8. doi: 10.1038/sj.bjc.6603717

40. Land LH, Dalton SO, Jorgensen TL, Ewertz M. Comorbidity and Survival After Early Breast Cancer. A Review. Crit Rev Oncol Hematol (2012) 81:196205. doi: 10.1016/j.critrevonc.2011.03.001

41. Land LH, Dalton SO, Jensen MB, Ewertz M. Impact of Comorbidity on Mortality: A Cohort Study of 62,591 Danish Women Diagnosed With Early Breast Cancer, 1990-2008. Breast Cancer Res Treat (2012) 131:1013-20. doi: 10.1007/s10549-011-1819-1

42. Tetsche MS, Dethlefsen C, Pedersen L, Sorensen HT, Norgaard M. The Impact of Comorbidity and Stage on Ovarian Cancer Mortality: A Nationwide Danish Cohort Study. BMC Cancer (2008) 8:31. doi: 10.1186/1471-2407-8-31

43. Jiao YS, Gong TT, Wang YL, Wu QJ. Comorbidity and Survival Among Women With Ovarian Cancer: Evidence From Prospective Studies. Sci Rep (2015) 5:11720. doi: 10.1038/srep11720

44. Post PN, Hansen BE, Kil PJ, Janssen-Heijnen ML, Coebergh JW. The Independent Prognostic Value of Comorbidity Among Men Aged $<75$ Years With Localized Prostate Cancer: A Population-Based Study. BJU Int (2001) 87:821-6. doi: 10.1046/j.1464-410x.2001.02189.x

45. Bradley CJ, Dahman B, Anscher M. Prostate Cancer Treatment and Survival: Evidence for Men With Prevalent Comorbid Conditions. Med Care (2014) 52:482-9. doi: 10.1097/MLR.0000000000000113

46. Post PN, Kil PJ, Hendrikx AJ, Janssen-Heijnen ML, Crommelin MA, Coebergh JW. Comorbidity in Patients With Prostate Cancer and Its Relevance to Treatment Choice. BJU Int (1999) 84:652-6. doi: 10.1046/ j.1464-410x.1999.00279.x

47. Fowler JE Jr, Terrell FL, Renfroe DL. Co-Morbidities and Survival of Men With Localized Prostate Cancer Treated With Surgery or Radiation Therapy. J Urol (1996) 156:1714-8. doi: 10.1016/S0022-5347(01)65489-2

48. Weinberger B, Herndler-Brandstetter D, Schwanninger A, Weiskopf D, Grubeck-Loebenstein B. Biology of Immune Responses to Vaccines in Elderly Persons. Clin Infect Dis (2008) 46:1078-84. doi: 10.1086/529197

49. Mazzola P, Radhi S, Mirandola L, Annoni G, Jenkins M, Cobos E, et al. Aging, Cancer, and Cancer Vaccines. Immun Ageing (2012) 9:4. doi: 10.1186/17424933-9-4

50. Koppie TM, Serio AM, Vickers AJ, Vora K, Dalbagni G, Donat SM, et al. AgeAdjusted Charlson Comorbidity Score Is Associated With Treatment Decisions and Clinical Outcomes for Patients Undergoing Radical Cystectomy for Bladder Cancer. Cancer (2008) 112:2384-92. doi: 10.1002/ cncr.23462

51. Sogaard M, Thomsen RW, Bossen KS, Sorensen HT, Norgaard M. The Impact of Comorbidity on Cancer Survival: A Review. Clin Epidemiol (2013) 5:3-29. doi: 10.2147/CLEP.S47150

52. Bebe FN, Hu S, Brown TL, Tulp OL. Role, Extent, and Impact of Comorbidity on Prognosis and Survival in Advanced Metastatic Melanoma: A Review. J Clin Aesthet Dermatol (2019) 12:16-23.

Conflict of Interest: The authors declare that the research was conducted in the absence of any commercial or financial relationships that could be construed as a potential conflict of interest.

Publisher's Note: All claims expressed in this article are solely those of the authors and do not necessarily represent those of their affiliated organizations, or those of the publisher, the editors and the reviewers. Any product that may be evaluated in 
this article, or claim that may be made by its manufacturer, is not guaranteed or endorsed by the publisher.

Copyright $\odot 2022$ Chang, Hsieh, Chen, Chu, Huang, Wang and Wei. This is an openaccess article distributed under the terms of the Creative Commons Attribution
License (CC BY). The use, distribution or reproduction in other forums is permitted, provided the original author(s) and the copyright owner(s) are credited and that the original publication in this journal is cited, in accordance with accepted academic practice. No use, distribution or reproduction is permitted which does not comply with these terms. 